\title{
Approche des laboratoires sur puce par l'expérimentation : projet MyLOC
}

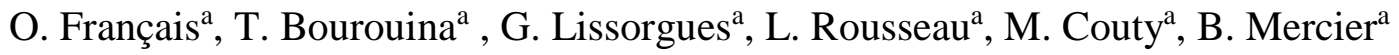 \\ a ESIEE-Paris, Département Santé Energie Environnement, Cité Descartes, Marne-la- \\ Vallée, France \\ Contact email : olivier.francais@esiee.fr
}

\begin{abstract}
Au sein de l'établissement ESIEE-Paris, nous avons mis en place des enseignements de niveau Master autour des laboratoires sur puce (Unité MyLOC : My Lab On Chip). Ces enseignements ont une approche type « learning by doing » avec l'utilisation de bureau d'étude où les élèves découvrent la thématique au travers de problématiques scientifiques et industrielles auxquelles ils doivent proposer des solutions. Cet article présente la partie microfluidique de ces enseignements (de la conception au test). Les élèves abordent d'abord la microfluidique sur la conception et analyse d'un circuit microfluidique afin de garantir son bon fonctionnement pour une application donnée (ici un test de Emmel sur puce pour la détection de cellules drépanocytaires). Les élèves apprennent ensuite à simuler son fonctionnement à l'aide d'un logiciel numérique multiphysique. Ils réalisent alors un prototype de puce en salle blanche et le testent afin de confronter les résultats aux modèles (analytique et numérique) mis en œuvre. Cette approche pédagogique très fortement orientée sur l'expérience a eu des retours très positifs. Cet enseignement a bénéficié de l'appui des Initiatives Pédagogique Innovantes de l'université Paris-EST (IDEA) et du CEMIP (Pôle parisien du CNFM).
\end{abstract}

\section{Le projet MyLOC : My Lab-On-Chip}

Le projet pédagogique «MyLOC » a pour contexte un enseignement dans le domaine des microsystèmes et des laboratoires sur puce (MyLOC : My Lab On Chip). C'est un enseignement qui a été mis en place à un niveau Master pour des élèves de la filière internationale «IME » (International Master of Electronics) (1) et de la filière Ingénieurs $5^{\text {ème }}$ année en Biotechnologie (2) au sein de l'établissement ESIEE-Paris.

La pédagogie utilisée est une approche par problèmes où l'enseignant est mis en position d'expert pour orienter et guider les élèves vers une solution répondant à la problématique. Au-delà de l'apprentissage d'un savoir et savoir-faire, l'enseignement vise à stimuler la capacité à travailler en groupe et à rendre compte d'un travail (Soft skills).

Le côté pluridisciplinaire des microsystèmes, et plus particulièrement des laboratoires sur puce (Microtechnologie, Instrumentation, Physique, Biologie,...), nous a amené à repenser l'enseignement afin que les élèves en aient une approche plus expérimentale que théorique avec un échange des informations qui soit dynamique.

Une plateforme de e-learning (Blackboard (C) est utilisée afin de mettre à disposition des élèves du contenu pédagogique (Polycopiés, Vidéos, Tutoriels, Datasheet, Procédés technologiques) (Figure 1). Selon la problématique à étudier (puce microfluidique (3)(4), 
capteurs pour l'eau (5), biocapteurs (6)), ils vont y trouver l'information pertinente, se l'échanger pour l'appliquer ensuite à leur étude de cas. Chaque étude de cas est associée à une réalisation dans les salles blanches de l'ESIEE-Paris dont le résultat sert ensuite de support à une caractérisation pour confronter l'étude théorique à la caractérisation pratique.

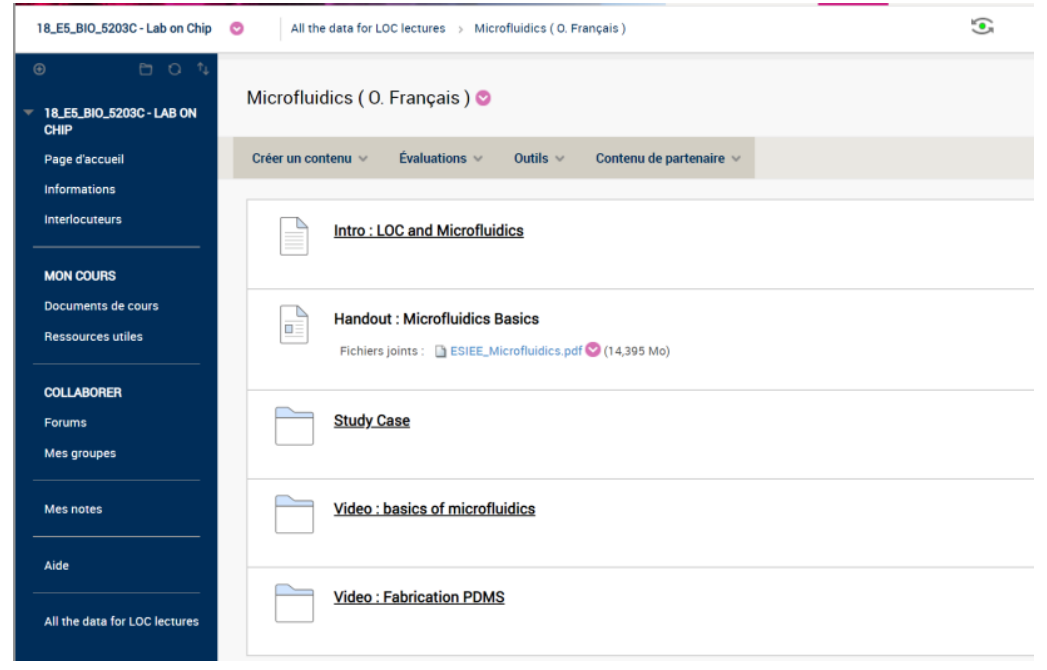

Fig.1. Capture d'écran de la plateforme d'e-learning Blackboard.

L'emploi du temps est adapté afin de dégager du temps de travail personnel non encadré sur lequel les étudiants effectuent leur apprentissage en mode bureau d'étude. Sur ces créneaux horaires, ils peuvent solliciter les enseignants comme « expert » afin d'éviter qu'ils ne soient dans une situation de blocage vis-à-vis des études qu'on leur demande de faire.

Sur la durée de l'unité 30h, les élèves suivent 10h de séances de cours/TD encadrés, 10h de séances de TP encadrés et $10 \mathrm{~h}$ de séances «travail personnel » où l'expert peut être sollicité mais n'est pas présent. Les élèves sont répartis en groupe de 5 à 6 élèves maximum ce qui permet de constituer des équipes de travail équivalentes à un petit bureau d'étude.

L'évaluation du module se fait au travers des réalisations des étudiants par groupe (rapports d'étude de cas, rapport de synthèse) et une présentation orale finale autour d'un thème dans le domaine des laboratoires sur puce. Il n'y a pas d'examen.

Pour mettre en place cet enseignement nous avons bénéficié d'un support financier de l'«IDEA » qui est une Initiative d'Excellence en Formations Innovantes (IDEFI) portée par huit établissements membres d'Université Paris-Est (UPE) (7) ainsi que du CEMIP (8) qui est le pôle Parisien du CNFM (9). Ce support financier a permis la mise en place d'équipements pour la caractérisation des puces microfluidiques, la mise en œuvre des enseignements sur la plateforme pédagogique et le développement des réalisations en salle blanche.

\section{La microfluidique par la pratique}

Sur la partie qui est consacrée à la microfluidique des laboratoires sur puce (gestion et maitrise des écoulements), la problématique du mélange et de la séparation est une des thématiques abordées. Trois phases ont été élaborées pour amener progressivement les élèves à s'approprier les connaissances et les mettre en pratique. Ces trois phases (Conception, réalisation puis caractérisation) s'enchainent tout au long des bureaux d'étude qui leur sont soumis. A la fin du module, ils en font un exposé global associé à un rapport de synthèse thématique. L'exposé global est mis en perspective d'un domaine d'application 
plus large mais qui fait appel aux éléments sur lequel ils ont acquis du savoir et du savoir faire lors des séances du module.

\section{a. Phase 1 : Conception / simulation}

Le contexte applicatif utilisé est celui de la maladie de la drépanocytose qui induit une rigidification des globules rouges (GRs) par polymérisation de l'hémoglobine lorsque les GRs sont désoxygénés, cela leur confère une forme de faucille. Il leur est demandé d'étudier cette pathologie puis de mettre un place une puce microfluidique qui permet de détecter la présence de GRs drépanocytaires à l'aide du test de Emmel.

Partant d'un concept de micro-mélangeur applicable au test de Emmel sur une puce microfluidique, les élèves posent les bases du calcul permettant de dimensionner le mélangeur (3), en s'appuyant sur les documents mis à leur disposition sur la plateforme de e-learning Blackboard et l'appui du professeur/expert. Cette phase de compréhension de la microfluidique et de conception leur fournit les outils théoriques permettant une première approche d'un écoulement microfluidique continu.

Les paramètres adimensionnels que sont le nombre de Reynolds $(\mathrm{Re})$ et le nombre de Péclet $(\mathrm{Pe})$ sont des éléments abordés et manipulés afin d'arriver à une solution aux problèmes posés (3). Dans le cadre de la microfluidique, où les écoulements sont laminaires $(\operatorname{Re}<1)$, ils utilisent l'analogie électrique-fluidique de manière à dimensionner l'architecture qui leur est demandée au travers du calcul des résistances hydrauliques (Figure 2).

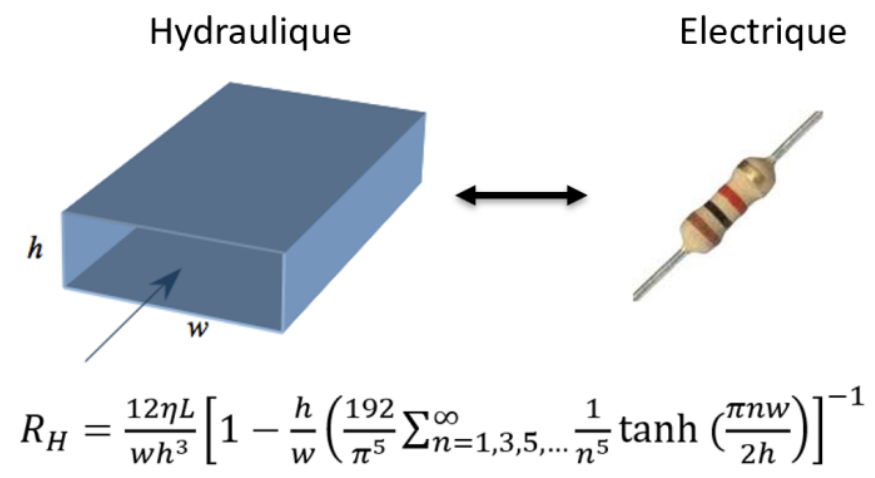

Fig.2. Représentation par une résistance électrique d'un canal microfluidique et expression de cette résistance hydraulique.

Le logiciel Comsol (C) est ensuite utilisé pour avoir une meilleure compréhension des écoulements en environnement microfluidique et ainsi valider par la simulation le fonctionnement d'un micro-mélangeur. On exploite une publication autour d'un filtre en $\mathrm{H}$ (Figure 3) qui illustre le phénomène de diffusion et de mélange pour faire de la séparation/mélange en environnement microfluidique selon la valeur de Pe (10). Cette étape met en œuvre de la simulation couplée entre de l'écoulement fluidique et du transport de matière au sein de micro-canaux. 


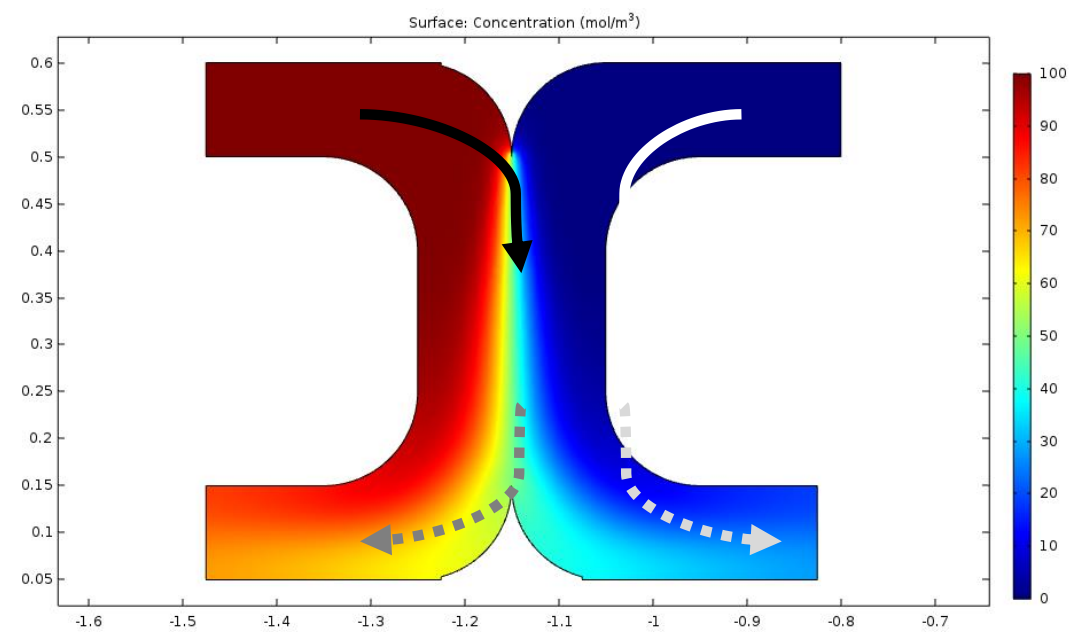

Fig.3. Illustration de la diffusion en environnement microfluidique. fond.

Cette phase donne lieu à deux rapports qui sont évalués autant sur la forme que sur le

\section{b. Phase 2 : Fabrication d'un puce microfluidique}

Une fois la partie conception/simulation validée, les élèves vont en salle blanche (ESIEE-Paris) pour mettre un œuvre un procédé complet de fabrication d'une puce microfluidique en PDMS (Poly-Diméthyl-Siloxane) (3). On s'appuie pour cela sur un masque qui contient un ensemble type de circuit microfluidique représentatif des laboratoires sur puce (Réseau de microcanaux, Filtre en $\mathrm{H}$, générateur de gradient, générateur de gouttes,...). Les étudiants mettent en œuvre la réalisation du moule par photolithographie de résine épaisse (SU8 2050) (11). Ce moule en relief sert alors de support pour réaliser les circuits microfluidiques en PDMS par moulage (Figure 4).

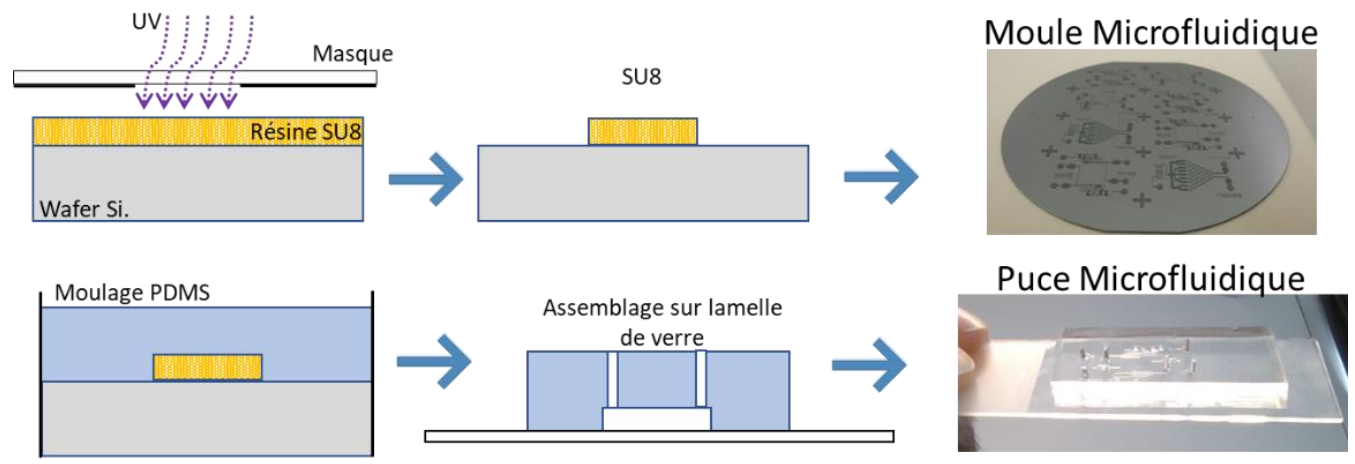

Fig.4. Procédé de réalisation en salle blanche des puces Microfluidique

Le PDMS est alors coulé sur le moule puis réticulé (Figure 4). Les étudiants effectuent en salle blanche le découpage des puces, le perçage des accès et le collage sur une lamelle de microscope par une activation plasma sous oxygène. 

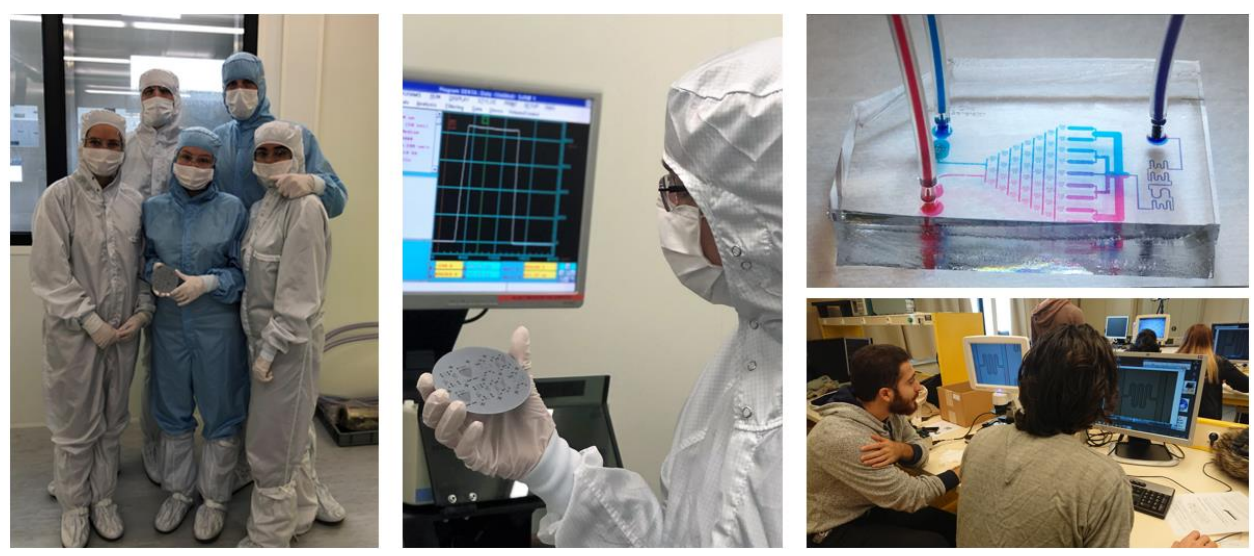

Fig.5. Fabrication en salle blanche d'une puce microfluidique et caractérisation des écoulements.

Cette fabrication en salle blanche dure 2 séances de travaux pratiques. Les élèves découvrent l'environnement salle blanche et les procédés de photolithographie. C'est aussi l'occasion de leur faire visiter l'ensemble des équipements et des composantes d'une salle blanche (Figure 5) en y incluant les équipements de retraitement de l'eau et de l'air.

Il n'est pas demandé de rapport sur cette phase. Par contre, on attend des élèves qu'ils y exploitent des éléments dans leur rapport de synthèse thématique.

\section{c. Phase 3 : Caractérisation d'une puce microfluidique}

Afin de faire le lien avec la conception et d'en avoir un retour, les étudiants caractérisent les puces qu'ils ont fabriquées et «packagées » en salle blanche. Ils mettent en œuvre sous vidéo-microscope les écoulements microfluidique par le biais de pousse-seringues qui sont reliés à la puce.

A partir des captures d'image et de l'utilisation du logiciel ImageJ (C, l'analyse des profils d'intensité de couleur au sein des canaux microfluidiques permet de caractériser la diffusion de colorants et d'estimer l'efficacité du mélange. En jouant sur les débits associés aux pousse-seringues, il est possible de faire évoluer le nombre de Peclet pour être expérimentalement dans des conditions de transport majoritaire par convection ou alors par diffusion afin de faire un lien avec la phase de conception (Figure 6).

Il n'est pas demandé de rapport sur cette phase. Par contre, on attend des élèves qu'ils intègrent des éléments de cette phase dans leur rapport de synthèse thématique.

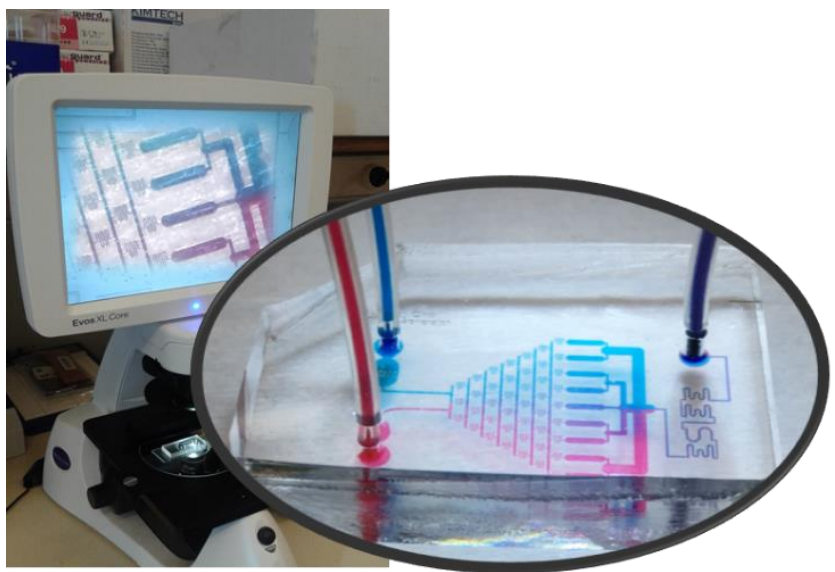

Fig.6. Caractérisation sous vidéo-microscope des écoulements microfluidique : cas d'un générateur de gradient. 
Ils ont ainsi une vision globale de l'élaboration d'une puce microfluidique. A partir de la caractérisation qui est faite, ils peuvent valider ou corriger la phase initiale de conception où certaines hypothèses simplificatrices peuvent avoir été faites.

\section{III.Evaluation de l'enseignement : retour des élèves}

Cet enseignement a fait l'objet d'une évaluation spécifique auprès des étudiants au travers d'un questionnaire dédié abordant 4 thèmes sur l'unité d'enseignement «MyLOC »:

- évaluation globale sur l'U.E. : Attentes et satisfactions,

- approche pédagogique de l'U.E.,

- niveau de difficulté et de compréhension,

- place dans leur apprentissage.

Cette enquête a mis en avant l'approche très concrète de l'enseignement où l'apprentissage par l'expérience permet de mettre en évidence les phénomènes de diffusion et mélange au sein d'une puce microfluidique. Certains étudiants font ressortir l'approche « chercheur » du module, où ils ont une certaine liberté de réponse vis-à-vis des sujets de bureaux d'étude sans connaissance préalable précise. Ce sentiment est renforcé par le travail en équipe où de manière effective durant les séances de travail personnel ils échangent et s'expliquent entre eux les solutions mises en œuvre. Cette liberté a un effet perturbant pour certains élèves qui ont du mal au début à comprendre les attendus mais aussi à estimer la charge de travail qui est plus lourde qu'un enseignement classique. Ce point ressort sur l'intensité du travail qu'ils fournissent qui lors de l'évaluation du module apparait plus important comparativement aux autres modules.

L'ensemble des étudiants ont eu le sentiment d'avoir mieux appris et avec plus d'intérêt que dans le cadre d'un enseignement classique. Ils ont apprécié d'être évalués sur ce qu'ils ont été capables de produire durant l'unité et qui reflète leur investissement. Le travail en groupe qui leur a été proposé a permis de lisser les difficultés, que l'on rencontre lorsque l'on découvre une thématique pluridisciplinaire, par l'appui que l'on peut trouver auprès des autres et des interactions qui en découlent. La place de l'expert est pour eux centrale et elle ressort comme une implication des professeurs mais aussi une découverte des travaux de recherche qu'ils mènent et qui sont ici en trame de fond du module.

Enfin, de manière unanime, la découverte de la salle blanche avec la possibilité de réaliser par eux-mêmes un «objet miniaturisé » dont ils ont au préalable fait un dimensionnement puis de le caractériser a été une grande satisfaction pour tous.

\section{Conclusion}

La mise en place de cet enseignement autour des laboratoires sur puce a permis de fédérer plusieurs professeurs et plateformes de l'ESIEE-Paris sur la même thématique mais abordant des applications différentes (Microfluidique, Biocapteurs, Monitoring de l'eau). La possibilité pour les élèves d'aller en salle blanche et de réaliser un circuit à l'aide des outils de la micro-électronique (dépôt de couches minces, photolithographie, gravure) a été l'élément principal de satisfaction pour eux.

L'enseignement a été mis en place afin d'avoir un équilibre entre l'aspect théorique, la conception/simulation et la mise en œuvre au travers de la fabrication/caractérisation de laboratoire sur puce. Les élèves sont évalués non pas au travers d'examen mais par la rédaction de rapports autour de bureaux d'études et de synthèses thématiques présentés à l'oral devant l'ensemble de la classe. Cela les met dans une position active forte tout au 
long du module et améliore la qualité de l'apprentissage et leur appropriation du contenu qui s'effectue fortement au travers de l'expérience. Cette approche pédagogique demande de leur part un investissement en temps et une charge de travail plus importante que pour un module classique type Cours/TD/TP/Examen.

Des outils de caractérisation ont été ainsi développés et utilisés pour cette approche pédagogique (plateforme microfluidique, suivi en température et en conductivité de milieu liquide en environnement confiné). Ils permettent désormais de proposer des moyens et des méthodes pour découvrir les microtechnologies appliquées ici dans un contexte de laboratoire sur puce.

\section{Remerciements}

Pour la mise en place de ce projet MyLOC au sein d'ESIEE-Paris, nous tenons à remercier l'IDEA (Initiative d'excellence en formations innovantes) de l'université Paris Est (7), IDEFI-FINMINA (Formation Innovante en Microélectronique et Nanotechnologies (12)) et le GIP-CNFM (Groupement d'Intérêt Public - Coordination Nationale pour la formation en Microélectronique et en nanotechnologies) (9) via le pôle parisien CEMIP (8) pour leur support financier. Nous remercions aussi le SMM (Service des Moyens de la Microélectronique) de l'ESIEE-Paris pour l'accueil et le support qu'ils ont apportés pour le bon fonctionnement de ce projet lors des séances en salle blanche.

\section{Références}

1. IME - International Master of Electronics., Website : https://www.esiee.fr/en/programmes/international-masters/master-of-electronics (Sept. 2018)

2. Filière Biotechnologie et e-Santé, Website https://www.esiee.fr/fr/formations/ingenieur/filieres/biotechnologies-esante (Sept. 2018)

3. Oh, K. W., Lee, K., Ahn, B., \& Furlani, E. P. (2012). Design of pressure-driven microfluidic networks using electric circuit analogy. Lab on a chip. https://doi.org/10.1039/c2lc20799k

4. Lu, X., Galarneau, M. M., Higgins, J. M., \& Wood, D. K. (2017). A microfluidic platform to study the effects of vascular architecture and oxygen gradients on sickle blood flow. Microcirculation (New York, N.Y. : 1994), 24(5). https://doi.org/10.1111/micc.12357

5. Shaun, F., Nefzaoui, E., Regina, H., Cesar, W., Marty, F., Capochichi-Gnambodoe, M., Bourouina, T. (2017). On the co-integration of a thermo-resistive flow-rate sensor in a multiparameter sensing chip for water network monitoring. In 2017 19th International Conference on Solid-State Sensors, Actuators and Microsystems (TRANSDUCERS) (pp. 1069-1072). IEEE. https://doi.org/10.1109/TRANSDUCERS.2017.7994237

6. Lim, J. W., Ha, D., Lee, J., Lee, S. K., \& Kim, T. (2015). Review of Micro/Nanotechnologies for Microbial Biosensors. Frontiers in Bioengineering and Biotechnology, 3. https://doi.org/10.3389/fbioe.2015.00061

7. IDEA - Initiative d'excellence en formations innovantes, Université Paris-Est, Website: http://idea.univ-paris-est.fr/fr (Sept. 2018)

8. CEMIP : Centre de Micro-Electronique de Paris Ile de France. Website : http://ingenierie.sorbonneuniversite.fr/fr/les-plateformes/le cemip.html (Septembre 2018)

9. GIP-CNFM: Groupement d'Intérêt Public - Coordination Nationale pour la formation en Microélectronique et en nanotechnologies. Website: http://wwww.cnfm.fr (Accès juillet 2018)

10. Brody, J. P., \& Yager, P. (1997). Diffusion-based extraction in a microfabricated device. Sensors and Actuators A: Physical, 58(1), 13-18. https://doi.org/10.1016/S0924-4247(97)80219-1

11. Picot, J., Ndour, P. A., Lefevre, S. D., El Nemer, W., Tawfik, H., Galimand, J., Français, O. (2015). A biomimetic microfluidic chip to study the circulation and mechanical retention of red blood cells in the spleen. American Journal of Hematology, 90(4), 339-345. https://doi.org/10.1002/ajh.23941

12. IDEFI-FINMINA : Initiative d'Excellence - Formation Innovante en MIcroélectronique et Nanotechnologies, ANR-11-IDFI-0017. Website: http://www.cnfm.fr/VersionFrancaise/actualites/ FINMINA.htm 
